\title{
THE ACYCLIC ELEMENTS OF A PEANO SPACE
}

\author{
A. D. WALLACE
}

We suppose throughout that $S$ is a Peano space. The notion of a cyclic element of such a space was introduced by G. T. Whyburn ${ }^{1}$ and $A$-sets were introduced independently by W. L. Ayres ${ }^{2}$ and Whyburn. Our present purpose is to consider a class of sets which may be regarded as the duals of cyclic elements.

Let $Q(S)$ denote the set of all cut-points and end-points of $S .^{3}$ Then each component of $Q(S)$ will be called an acyclic element of $S$. If $p$ and $q$ are two points of $S$ then we write $p \sim q$ to mean that no point separates p from $q$ in $S$. A set will be termed acyclic if it contains no simple closed curve, cyclic if each pair of points is on a simple closed curve of the set.

(i) Each cyclic element [acyclic element] is a cyclic [acyclic] A-set.

Proof. We prove only the second statement. Let $F$ be an acyclic element, $x_{n} \in F$ and suppose that $x_{n} \rightarrow x$. If $x$ is not in $F$ then since $F+x$ is connected we must have $x \in S-Q(S)$, from the definition of $F$ as a component of $Q(S)$. Consequently $x$ is a point of a cyclic element $E$ of $S$. If $(F+x) \cdot E$ contained only the point $x$ then it would follow that $F$ was contained in a single complementary domain $R$ of $E$ and thus $x=F(R)=\bar{R}-R$. Consequently $x$ would be a cut-point, which is obviously impossible. We conclude that $(F+x) \cdot E$ is a nondegenerate connected set. It is clear that no point of this set is an end-point and hence the set contains uncountably many cut-points. But no cyclic element contains more than a countable number of cut-points. We conclude that $x \in F$ and hence $F$ is closed. If $F$ is not an $A$-set there is an arc $p x q$ which meets $F$ in the set $p+q$. It is manifest that no point separates $p$ from $q$ in $S$ since $F$ is connected. That is, $p \sim q$ and hence $p+q \subset E$, a true cyclic element. By the argument given

${ }^{1}$ See Kuratowski and Whyburn, Fundamenta Mathematicae, vol. 16 (1930), p. 305. By a cyclic element we understand a nondegenerate set such that any two points lie on a simple closed curve and which is maximal relative to this property. This is not the definition given by Kuratowski and Whyburn but is equivalent (cf. G. T. Whyburn, this Bulletin, vol. 38 (1931), p. 429 and references given there) and is in fact Whyburn's original definition. It seems more natural in the present setting.

${ }^{2}$ See W. L. Ayres, this Bulletin, vol. 46 (1940), p. 794, for references. An $A$-set is a closed arc set.

${ }^{3}$ The terms cut-point and end-point without qualification refer to the space $S$. The proofs of all the statements concerning cyclic elements will be found in Kuratowski and Whyburn. We assume considerable familiarity with this fundamental work. 
above $E$ would contain uncountably many cut-points, a contradiction. Thus $F$ is an $A$-set. Since each $A$-set is a Peano space it is easy to see that $F$ contains no simple closed curve.

(ii) In order that a set $X$ of $S$ be a cyclic element [acyclic element] it is necessary and sufficient that it be a connected set which is maximal relative to the property: If $p$ and $q$ are points of $X$ then $p \sim q[p$ non $\sim q]$.

Proof. We prove only the second statement. Let $F$ be an acyclic element of $S, p, q \in F$, and let $t$ be the unique arc from $p$ to $q$ in $F$. Since $F$ is a tree there is a point $x$ on $t$ which separates $p$ from $q$ in $F$. But since $F$ is an $A$-set we know that $x$ separates $p$ from $q$ in $S$ so that we have $p$ non $\sim q$. Let $F^{\prime}$ be a connected set which contains $F$ and which further contains at least one point which does not belong to $F$. Because $F^{\prime}$ is connected it contains a point $p$ of $S-Q(S)$ and hence a point of $E$, a cyclic element. It is easy to see that if $F^{\prime}$ and $E$ have in common only the point $p$ then $p$ must be a cut-point, which is impossible since $p \in S-Q(S)$. Hence $F^{\prime} \cdot E$ contains another point $q$. But since $p, q \in E$ we have $p \sim q$ and by assumption $p$ non $\sim q$ because $p, q \in F^{\prime}$. Thus $F=F^{\prime}$. The condition of the theorem is thus necessary. It is not hard to see that the condition is sufficient.

(iii) No point of a cyclic element $E$ is an end-point or a cut-point of $E$ while every point of an acyclic element $F$ is either an end-point or a cut-point of $F$.

We use the term element indiscriminately for cyclic or acyclic element.

(iv) The elements of $S$ form a null sequence in the sense that for any positive number $\delta$ there are only finitely many whose diameters exceed $\delta$.

Proof. The result is known for cyclic elements. If there existed an infinite sequence of acyclic elements with diameters bounded from zero we could find an infinite subsequence with the same property and which converged to a nondegenerate continuum, that is, we have $F_{n} \rightarrow X, \delta(X) \geqq d>0$. If $a, b \in X$ and $a$ non $\sim b$, then we may write $S=M+N$, where $a \in M, b \in N, M \cdot N=p$, a cut-point. For all large $n$ it follows that $F_{n}$ meets both $M$ and $N$ and hence $p \in F_{n}$, contrary to the fact that $F_{i} \cdot F_{j}=0$ for $i \neq j$. We conclude that $X \subset E$, a cyclic element. It is easy to see that we can find two points $u, v \in X-\sum F_{n}$, since each acyclic element meets $E$ in at most one point and the set $X$ is uncountable. For $n$ sufficiently large we can find arcs $u u^{\prime}$ and $v v^{\prime}$ which are disjoint and such that $F_{n} \cdot u u^{\prime}=u^{\prime}, F_{n} \cdot v v^{\prime}=v^{\prime}$. Since $F_{n}$ is an $A$-set we can find an $\operatorname{arc} u^{\prime} v^{\prime}$ in $F_{n}$. Further we can find an arc $u v$ 
in $E$. The set $u v+u^{\prime} v^{\prime}+u u^{\prime}+v v^{\prime}$ contains a simple closed curve $J$ which has two points in $F_{n}$. Since we may suppose that $J$ meets $E$ in $u+v$ we must have $J \subset E$ and thus $F_{n}$ meets $E$ in more than one point.

(v) A Peano space is the sum of its elements and no two have more than one point in common. No two acyclic elements meet.

(vi) The decomposition of $S$ into acyclic elements and points of $S-Q(S)$ is upper semi-continuous. Thus the associated monotone transformation $T S=S^{\prime}$ of $S$ onto the hyper-space $S^{\prime}$ is topological on each cyclic element of $S$ and the acyclic elements of $S^{\prime}$ are all degenerate.

The results stated do not exhaust the list of dual properties but we have tried to give the most striking. It appears improbable that the idea could be extended to non-locally connected spaces using the definitions given by Kelley, ${ }^{4}$ Moore,${ }^{5}$ or Whyburn $;{ }^{6}$ or to the generalizations due to Hall ${ }^{7}$ and Youngs. ${ }^{8}$

The UNIVERsity OF Virginia AND

Princeton University

${ }^{4}$ J. L. Kelley, Proceedings of the National Academy of Sciences, vol. 26 (1940), p. 192.

${ }^{5}$ R. L. Moore, Foundations of Point Set Theory, American Mathematical Society Colloquium Publications, vol. 13, New York, 1932.

6 G. T. Whyburn, American Journal of Mathematics, vol. 56 (1934), p. 133.

7 D. W. Hall, Transactions of this Society, vol. 47 (1940), p. 305.

8 J. W. T. Youngs, American Journal of Mathematics, vol. 52 (1940), p. 449. 